\title{
CLINICAL AND SEROLOGICAL PARAMETERS OF PROGRESSION AND PROGNOSIS IN PATIENTS WITH SYSTEMIC SCLEROSIS - A STATE OF THE ART REVIEW
}

DOI: 10.36740/WLek202007140

\author{
Ewa Wielosz, Maria Majdan \\ DEPARTMENT OF RHEUMATOLOGY AND CONNECTIVE TISSUE DISEASES, MEDICAL UNIVERSITY OF LUBLIN, LUBLIN, POLAND
}

\begin{abstract}
Systemic sclerosis (SSc) is a multi-organ connective tissue disease that leads to the dysfunction and the impaired morphology of blood vessels due to non-specific inflammation and progressive fibrosis. Due to the diversity of SSC and even though the factors predisposing to the severe course of SSC are known, it is not always possible to predict the disease progression and to determine the prognosis. Ideally, the group of patients with faster progression of organ lesions and a worse course of the disease should be identified and the early intensive treatment should be instituted.

The aim of the article, is an attempt to identify the factors that worsen the prognosis in the course of SSC.

The analysis of numerous studies demonstrated that patients with short-lasting SSc, with the presence of anti-RNA polymerase III antibodies, with a generalized type of SSc with quickly progressing skin lesions and males should be most strictly monitored. Moreover, vascular complications, tendon ruptures and fast capillaries loss observed in nailfold capillaroscopy are the factors deteriorating the prognosis in SSC.

In conclusion, despite the known, the factors that worsen the prognosis, it is difficult to predict the course of systemic sclerosis. Due to its incompletely elucidated etiopathology as well as the diverse and unpredictable nature of the disease, reliable markers to determine the prognosis in SSc have not been found.
\end{abstract}

KEY WORDS: systemic sclerosis, a course of the disease, poor prognostic factors

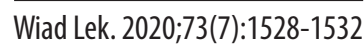

\section{INTRODUCTION}

Systemic sclerosis (SSc) is a multi-organ disease that belongs to systemic connective tissue diseases. The etiopathogenesis of the disease is not fully understood, although in early stages endothelial damage occurs with the disturbance in the morphology and the function of small blood vessels, inflammatory process and activation of the immune system and fibroblasts. The consequence of these processes is the damage to the vessels with progressive fibrosis of the skin and internal organs, especially the lungs, heart, digestive tract and kidneys. The course of SSc is very diverse, therefore in many cases we are not able to predict the consequences of the disease, and the degree of internal organ involvement as well as to determine the prognosis. The beginning of SSc is also heterogeneous. There is a group of patients with a long-term stable disease from the beginning of its onset, in whom no serious organ symptoms develop. On the other hand, the course can be very progressive. Then in a short time after or at the same time with the appearance of first symptoms of the disease, severe internal organ involvement occurs, even with sudden cardiac death or severe respiratory failure. Canadian physician William Osler who lived in 1849-1919 called SSc "one of the most terrible of all human ills" [1].

\section{Adverse prognostic factors in systemic sclerosis}

Despite the known factors indicating a more severe SSc course, we are not always able to predict the progression of the disease and to determine the prognosis [2]. Based on the literature data $[2,3]$, the known factors of the poor prognosis of SSc include:

- generalized type of the disease,

- early involvement of internal organs, especially lungs, heart or kidneys,

- high inflammatory parameters

- male gender.

On the other hand, a milder course of the disease can be demonstrated by:

- limited type of SSc,

- absence or benign involvement of internal organs,

- presence of anti-centromere antibodies

- overlap SSc with other autoimmune diseases.

The optimal strategy would be to identify a group of patients who will have a faster progression of organ changes and a worse course of the disease, and to implement early intensive treatment in this group (Table 1]).

\section{THE AIM}

The aim of the article, is an attempt to identify the factors that worsen the prognosis in the course of SSc. 
Table 1. Analysis of the significance of selected prognostic factors in systemic sclerosis

\begin{tabular}{ccc}
\hline Prognostic factor & Worse prognosis & Better prognosis \\
\hline Demographics & Male sex & Female sex \\
\hline SSc type & Generalized & Limited \\
\hline Presence of antibodies & Anti-RNA polymerase III antibodies & Normal ESR and CRP \\
\hline Inflammation parameters & High ESR or CRP & Slow skin lesion progression \\
\hline Skin involvement & Fast skin lesions progression & FVC $>70 \%$ \\
\hline Internal organs involvement & Early involvement of lungs, heart and \\
kidneys or tendons rupture & Do or molvement of internal organs $>80 \%$ \\
\hline Ligh resolution computed tomography & Pulmonary lesions extension $>20 \%$ & Pulmonary lesions extension $<10 \%$ \\
\hline Vascular complications - fingertips ulcers & Fingertip ulcerations & No fingertip ulcerations \\
\hline Capillaroscopy & Fast capillaries loss & No or slow capillaries loss \\
\hline
\end{tabular}

FVC - forced vital capacity, DLCO - [\%] predicted diffusing capacity for carbon monoxide, CRP - C-reactive protein, ESR - erythrocyte sedimentation rate

\section{REVIEW AND DISSCUSION}

Is it possible to predict the course of the disease based on the assessment of skin lesions?

Harrick et al. in their research evaluated patients with early generalized SSc with rapid progression of skin lesions and attempted to predict the course of the disease during 24 months of follow-up [3]. In a group of 326 patients with SSc, the degree of skin involvement was assessed every 3 months. The extent of skin lesions was determined by means of skin fold thickness assessment, defined as Rodnan skin score (RSS) [4]. The skin fold thickness was assessed by palpation on a scale of 0 to 3 points in 17 different areas of the body. An increase by $25 \%$ and more than 5 points in RSS was considered to be a progression risk factor. The authors of the study showed that during 24 months of follow-up RSS increased by $25 \%$ in $22.5 \%$ of patients. This group of patients characterized a shorter duration of the disease and a lower RSS at the beginning of the study [3]. The study also analyzed a group with the presence of anti-RNA polymerase III antibodies and found that more than $29 \%$ of patients with these antibodies had a $25 \%$ increase in RSS over the 2 years of follow-up. It was also shown that this group with the presence of anti-RNA polymerase III antibodies had the highest RSS at the start of the study, the highest maximum rate of skin involvement during follow-up compared to the group of patients with SSc without the presence of anti-RNA polymerase III antibodies. Summing up the study, authors showed that the group of patients with SSc who baseline had a lower RSS and a shorter duration of the disease presented a greater progression of skin lesions during the 24 months of follow-up. This indicates that the baseline value of RSS is not a sufficient prognostic risk factor. In addition, patients with a short duration of the disease and with the presence of anti-RNA polymerase III antibodies should be closely monitored [3].

\section{MORTALITY IN SYSTEMIC SCLEROSIS}

Hao et al. examined a group of 1,070 patients with SSc lasting less than 4 years and compared with a group of
3,218 patients with SSc lasting longer than 4 years [2]. The mortality rate, life expectancy and age at death were assessed in both groups. It was shown that the mortality rate was higher in the group of patients with SSc with a shorter duration of the disease, and especially in men with a shorter duration of the disease. It was also shown that the mean life expectancy in the group of patients with a shorter duration of the disease was lower. In addition, in the group of SSc patients with a duration of less than 4 years, there were 140 deaths, of which over $60 \%$ were deaths associated with SSc, and interstitial lung disease or pulmonary arterial hypertension were the main causes. Cancer, sepsis, cerebrovascular disease and ischemic heart disease dominated among causes of non-SSc deaths. Analyzing a group of patients with a longer duration of the disease, 440 deaths were recorded, of which over $50 \%$ were associated with SSc. It was shown that risk factors for death in the group of patients who had the disease for less than 4 years were male sex, more advanced age at the time of diagnosis, generalized SSc, pulmonary arterial hypertension and scleroderma renal crisis, while a longer duration of the disease was the protective factor indicating a better prognosis in this group. In the population of patients with SSc, whose disease duration exceeded 4 years, the risk factors for death were also male sex, more advanced age at the time of diagnosis, generalized SSc, pulmonary arterial hypertension and, in addition, interstitial lung disease, while longer duration of the disease and the presence of anti-centromere antibodies were factors associated with better prognosis. To sum up this research, early mortality in SSc is significant, especially in the group of patients with the duration of the disease is less than 4 years, and especially in males and in case of generalized SSc $[2,5]$.

Can the prognosis of interstitial lung disease in the course of systemic sclerosis be predicted?

Predicting the course of SSc in patients with interstitial lung disease is very challenging. Already in 2008 Goh et al. demonstrated the relationship between the extent of lung lesions assessed in high resolution computed tomography 


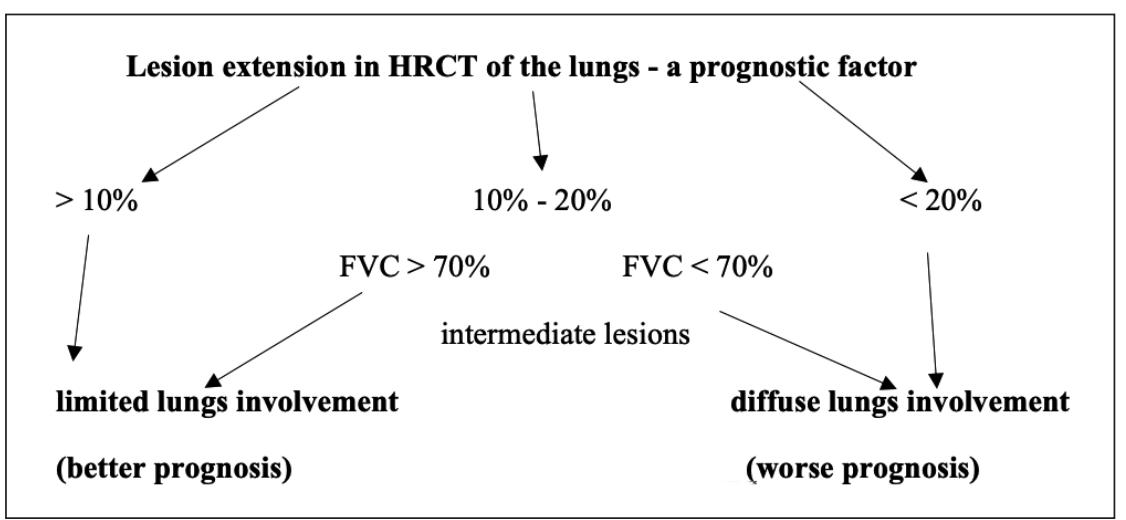

Fig. 1. Prognosis in interstitial lung disease in systemic sclerosis based on the assessment of the extent of pulmonary lesions in HRCT [6]. HRCT - high resolution computed tomography
(HRCT) and the prognosis in interstitial lung disease associated with SSc [6]. Authors revealed that in case of pulmonary lesions below $10 \%$ in lung HRCT, there is limited lung involvement and a better prognosis. In case of lung involvement above $20 \%$ with diffuse lung involvement authors disclosed a worse prognosis. Pulmonary changes between $10 \%-20 \%$ in lung HRCT are considered intermediate and then pulmonary function tests are important. In case of intermediate pulmonary changes, if forced vital capacity (FVC) is above $70 \%$, then the prognosis is better, and $\mathrm{FVC}$ below $70 \%$ is associated with a worse prognosis (Fig. 1) [6].

In 2017, Moriset et al. assessed the risk of death during the first 3 years of SSc duration with associated interstitial lung disease based on the SADL model (S - ever smoking history, A - age, DL - DLCO [\%] predicted diffusing capacity for carbon monoxide) [7]. The study involved 225 patients with interstitial lung disease in the course of SSc and based on the SADL model, the percentage of patients with a low, medium and high risk of death was determined. $21 \%$ of patients had a high score calculated on the basis of the SADL model. The highest risk of death was found in the group of patients with high scores in the SADL model [7]. In addition, smoking, more advanced age and rapid decline of DLCO were shown to be death risk factors in the first years of SSc with interstitial lung disease. To sum up, on the basis of the SADL model it is possible to distinguish a group of patients with SSc with interstitial lung disease and with a high risk of death during the first 3 years of the disease, and to some extent to predict the prognosis in this group of patients [7]. In another research, Wu et al. showed that a decrease in saturation below $94 \%$ during a 6-minute walk test and a history of arthritis were independent risk factors for the progression of interstitial lung disease in the course of SSc during one-year follow-up [8]. In 2019, a large multicenter study of the EUSTAR group - European Scleroderma Trials and Research Study Group - covering over 1,000 patients was published. It revealed that the progression of skin lesions within one year were associated with worsening of lung function tests and worse survival in patients with generalized SSc $[9,10]$.

Relationship between vascular changes and prognosis in systemic sclerosis

Many studies were conducted to determine the relationship between vascular changes and joint symptoms in predicting the course of the disease and assessing the prognosis in patients with SSc. One of the large multicenter studies conducted by the EUSTAR group evaluated over 3,000 patients with SSc for the presence of ulcers on fingertips. Fingertip ulcerations (present or in history) were found in $34 \%$ of patients. Authors demonstrated that the history of fingertip ulcers was a predictive factor for the development of new ulcers, cardiovascular complications, including pulmonary arterial hypertension and shortened life expectancy [11]. Another study of the EUSTAR group focused on the association of musculoskeletal symptoms with the prediction of SSc progression. Arthritis and tendon rupture were found to be independent factors in the progression of skin lesions in the course of SSc. In addition, arthritis was a predictor of new fingertip ulcers and worsening of left ventricular ejection fraction. In addition, tendon ruptures were shown to be independent risk factors for the development of scleroderma renal crisis. In conclusion, arthritis and tendon rupture were independent predictors of the disease progression in the early phase of SSc [12]. A number of studies was also carried out to assess whether, based on abnormal nail-fold capillaries on capillaroscopy examination, it was possible to predict the degree of internal organ involvement and determine the prognosis. Smith et al. showed that capillaroscopic abnormalities of "late" scleroderma microangiopathy were associated with more frequent future development of vascular lesions and pulmonary complications during 2-year follow-up [13]. Authors showed that $63 \%$ of patients with SSc who at the time of enrollment had the image of "late" scleroderma microangiopathy with a significant reduction in the number of capillaries developed peripheral vascular complications, and as much as $73 \%$ of pulmonary complications within 18-24 months of follow-up [13, 14]. On the basis of a meta-analysis carried out by Paxton et al., a relationship was found between capillary loss in capillaroscopy examination of nail-fold capillaries and organ complications, in particular vascular disorders such as finger ulcers, development of pulmonary arterial hypertension, as well as the occurrence of calcinosis [15]. In summary, based on the progression of capillaroscopic changes, one can also predict the degree of internal organ involvement and determine the prognosis in SSc. In addition, it was also shown that some biomarkers such as: growth differentiation factor- 15 
(GDF-15), placenta growth factor (PGF), endostatins, and vascular-endothelial growth factor (VEGF) present in high serum concentrations in patients with SSc are factors increasing the risk of developing a generalized SSc subtype, interstitial lung disease, pulmonary arterial hypertension or fingertip ulceration [16].

Treatment of systemic sclerosis depending on the course and phase of the disease

Identifying adverse prognostic factors in the course of SSc is of particular importance in therapeutic management. It still remains a dilemma whether to intensively treat with immunosuppressive drugs patients with very early SSc, without involvement of internal organs. At a very early stage of the disease, close observation and monitoring of patients with early involvement of internal organs is suggested to identify those in the window of opportunity of therapeutic options and to treat them aggressively so as not to lead to irreversible changes in the course of the disease. One prospective study enrolled 326 patients with an early SSc phase from 50 centers and patients were followed up for 2 years. Patients were divided into four groups. The first group was treated with methotrexate 20-25 $\mathrm{mg}$ /week p.o., the second group - with mycophenolate mofetil $2 \times 1 \mathrm{~g} /$ day p.o., the third group - with cyclophosphamide $500 \mathrm{mg} / \mathrm{m}^{2} 1 \mathrm{x}$ month i.v. for 6-12 months or p.o. $1-2 \mathrm{mg} / \mathrm{kg} /$ day p.o. Methotrexate, mycophenolate mofetil or azathioprine were used as a continuation of the therapy. The fourth group was not treated with immunosuppressive drugs. In the study, no statistically significant differences were found between the four groups, however, not statistically significant improvement was shown in patients treated with immunosuppression. In addition, RSS improved in all four groups, however the smallest improvement was obtained in the group of patients not treated with immunosuppression. In that group there was also a higher mortality rate [17].

\section{CONCLUSIONS}

In summary, internal organ involvement in SSc often occurs early in the disease and may be associated with increased mortality. Factors that worsen the prognosis in the course of the disease include:

- male sex,

- a shorter duration of the disease,

- generalized SSc,

- the presence of organ complications such as pulmonary arterial hypertension, interstitial lung disease, fingertip ulceration or tendon rupture.

Factors that improve prognosis in systemic sclerosis include: - a longer duration of the disease,

- limited SSc,

- presence of anti-centromere antibodies.

The initial high RSS is not always a sufficient prognostic factor for a worse course of the disease, however, rapid progression of skin lesions is still associated with the risk of developing interstitial lung disease. Risk factors for death in interstitial lung disease during the first years of SSc are history of smoking, more advanced age and rapid decrease in DLCO. Changes in the capillaroscopy examination of the nailfold, especially early capillary loss, correlate with the progression of organ changes, in particular vascular complications and the development of interstitial lung disease in SSc. Despite the generally known factors of poor prognosis, in many cases it is difficult to predict the course of SSc and we are still not able to isolate sufficient markers that would allow an unambiguous assessment of the prognosis.

\section{REFERENCES}

1. Bellando-Randone S, Matucci-Cerinic M. Very Early Systemic Sclerosis and Pre-systemic Sclerosis: Definition, Recognition, Clinical Relevance and Future Directions. Curr Rheumatol Rep. 2017;19:65.

2. Hao Y, Hudson M, Baron M, et al. Early Mortality in a Multinational Systemic Sclerosis Inception Cohort. Arthritis Rheumatol. 2017;69:10671077.

3. Herrick AL, Peytrignet S, Lunt M, et al. Patterns and predictors of skin score change in early diffuse systemic sclerosis from the European Scleroderma Observational Study. Ann Rheum Dis. 2018;77:563-570.

4. Kowal-Bielecka 0, Domysławska I, Sierakowski S. Ocena zmian skórnych u chorych na twardzinę układową: uwagi praktyczne i znaczenie kliniczne. Reumatologia 2005; 43:310-312.

5. Strickland G, Pauling J, Cavill C, et al. Mortality in systemic sclerosis-a single centre study from the UK. Clin Rheumatol. 2013;32:1533-1539

6. Goh NS. Interstitial lung disease in systemic sclerosis: a simple staging system. Am J Respir Crit Care Med. 2008;177:1248-1254.

7. Morisset J, Vittinghoff E, Elicker BM, et al. Mortality Risk Prediction in Scleroderma-Related Interstitial Lung Disease: The SADL Model. Chest. 2017;152:999-1007.

8. WuW1, Jordan S, Becker M0, et al. Prediction of progression of interstitial lung disease in patients with systemic sclerosis: the SPAR model. Ann Rheum Dis. 2018;77:1326-1332.

9. Wu W, Jordan S, Graf N, et al. EUSTAR Collaborators. Progressive skin fibrosis is associated with a decline in lung function and worse survival in patients with diffuse cutaneous systemic sclerosis in the European Scleroderma Trials and Research (EUSTAR) cohort. Ann Rheum Dis. 2019;78:648-656.

10. Jhajj A, Gill HP, Hague CJ, et al. Pulmonary physiology is poorly associated with radiological extent of disease in systemic sclerosis-associated interstitial lung disease. Eur Respir J. 2019;2:53.

11. Mihai C, Landewé $R$, van der Heijde D, et al. Digital ulcers predict a worse disease course in patients with systemic sclerosis. Ann Rheum Dis. 2016;75:681-686.

12. Avouac J, Walker UA, Hachulla E, et al. Joint and tendon involvement predict disease progression in systemic sclerosis: a EUSTAR prospective study. Ann Rheum Dis. 2016;75:103-109.

13. Smith V, Decuman S, Sulli A, et al. Do worsening scleroderma capillaroscopic patterns predict future severe organ involvement? a pilot study. Ann Rheum Dis. 2012;71:1636-1639.

14. Smith V, Riccieri V, Pizzorni C, et al. Nailfold capillaroscopy for prediction of novel future severe organ involvement in systemic sclerosis. J Rheumatol. 2013;40:2023-2028.

15. Paxton D, Pauling JD. Does nailfold capillaroscopy help predict future outcomes in systemic sclerosis? A systematic literature review. Semin Arthritis Rheum. 2018;48:482-494.

16. Kawashiri SY, Nishino A, Igawa T, et al. A. Prediction of organ involvement in systemic sclerosis by serum biomarkers and peripheral endothelial function. Clin Exp Rheumatol. 2018;36 Suppl 113:102-108. 
17. Herrick AL, Pan X, Peytrignet S, et al Treatment outcome in early diffuse cutaneous systemic sclerosis: the European Scleroderma Observational Study (ESOS). Ann Rheum Dis. 2017;76:1207-1218.

\section{ORCID and contributionship}

Ewa Wielosz - 0000-0003-2193-7778 A,B,D,E,F

Maria Majdan - 0000-0002-4345-1675 A,B,D,E,F

\section{Conflict of interest}

Authors declare no conflict of interest.

\section{CORRESPONDING AUTHOR}

\section{Ewa Wielosz}

Department of Rheumatology and Connective Tissue Diseases,

Medical University of Lublin

Jaczewskiego Street 8; 20-607 Lublin,

e-mail: ewa.wielosz@wp.pl

Received: 08.03 .2020

Accepted: 29.05 .2020

A - Work concept and design, B - Data collection and analysis, C - Responsibility for statistical analysis,

$\mathbf{D}$-Writing the article, $\mathbf{E}$-Critical review, $\mathbf{F}$ - Final approval of the article 